Research Article

\title{
Computerized Tomography Imaging Omics under Iterative Reconstruction Algorithm in Diagnosis of Gastric Cancer
}

\author{
Chunfang Zhou $\mathbb{D}^{1},{ }^{1}$ Shufang Tian $\mathbb{D}^{2},{ }^{2}$ Fei Lv $\mathbb{D},{ }^{1}$ Rui Shang $\mathbb{D},{ }^{1}$ and Xuejiao Zheng $\mathbb{D}^{3}$ \\ ${ }^{1}$ Department of Gastroenterology, Renmin Hospital, Hubei University of Medicine, Shiyan 442000, Hubei, China \\ ${ }^{2}$ Department of Traditional Chinese Medicine, Renmin Hospital, Hubei University of Medicine, Shiyan 442000, Hubei, China \\ ${ }^{3}$ Department of Pharmacy, Renmin Hospital, Hubei University of Medicine, Shiyan 442000, Hubei, China
}

Correspondence should be addressed to Xuejiao Zheng; 20150700096@m.scnu.edu.cn

Received 19 September 2021; Revised 12 November 2021; Accepted 15 November 2021; Published 24 December 2021

Academic Editor: M Pallikonda Rajasekaran

Copyright (c) 2021 Chunfang Zhou et al. This is an open access article distributed under the Creative Commons Attribution License, which permits unrestricted use, distribution, and reproduction in any medium, provided the original work is properly cited.

\begin{abstract}
This study aimed to explore the application value of computed tomography (CT) imaging radiomics based on a sinogram-affirmed iterative reconstruction algorithm (SAFIRE) in the diagnosis of gastric cancer. 59 patients who were clinically diagnosed with gastric cancer were selected as research objects and arranged CT examinations. The images obtained were optimized by the SAFIRE for the staging of gastric cancer. The pathological biopsy results were used as the gold standard to evaluate its diagnostic effect and compared with the filtered back-projection (FBP) method. The results showed that the carrier-to-noise ratio (CNR) (0.979) and signal-to-noise ratio (SNR) (0.967) of the CT image after the algorithm processing were significantly higher than those $(0.781,0.744)$ before $(P<0.05)$. There was no significant difference in CT values between the FBP algorithm and S1, S2, and S3 $(P>0.05)$; the area under the curve (AUC) $(0.999)$ and sensitivity $(0.98)$ of the CT training group under the SAFIRE algorithm for gastric cancer classification were higher than those of the verification group $(0.958,0.92)$. The preoperative CT staging kappa value was consistent with the postoperative pathological diagnosis of 0.882 . CT images guided by SAFIRE can objectively and noninvasively assess the tumor asymmetry, discover additional information from subjective evaluation beyond the naked eye, and perform reasonable staging diagnosis of gastric cancer, which was useful for clinicians to develop high-quality individualized treatment plans.
\end{abstract}

\section{Introduction}

Gastric cancer is a common disease with high morbidity and mortality in China, which is common in the gastrointestinal tract and primary malignant tumors. East Asia is an area with high incidence of occult, and endoscopic screening is not yet widespread in China. The discovery was late, and the prognosis was poor $[1,2]$. There are a variety of factors that affect the outcome of patients. The degree of histological differentiation is an important factor. The lower the degree of differentiation, the higher the degree of malignancy, and the worse the prognosis. Decourcy et al. found that gastric cancer with different degrees of differentiation had different biological behavior and chemotherapy sensitivity. The symptoms of early gastric cancer are not obvious. The excision rate of early gastric cancer is high, up to $90 \%$. Once in the late stage, it develops rapidly. At present, surgical resection is the main treatment for gastric cancer [3-5]. Radical surgery and palliative surgery are the main methods of surgery. Radical surgery is the main treatment for early gastric cancer. Palliative surgery is adopted when the tumor cannot be completely excised in advanced gastric cancer. The choice of business scope and business mode has a great impact on the life of patients because the stomach plays a very important role in the entire digestive system. In addition to the production of gastric juice and digestive enzymes, there are many kinds of internal factors such as the secretion of functional cells and parietal cells, which are important factors in the production of red blood cells $[6,7]$. The choice of operating range may affect the blood system. Therefore, the early diagnosis of gastric cancer and the extent of tumor resection are very important. 
Computerized tomography (CT) is a common method for detecting gastric cancer [8]. It is very important to reduce the dose of radiation received by patients. At present, the main ways to reduce radiation dose include reducing tube voltage, tube current, increasing pitch, and reducing scanning times. Using low tube voltage scanning can improve the definition of iodine with high resolution [9]. Compared with traditional single-energy CT, dual-source CT scanning can obtain data and image fusion information under different voltages. The vast majority of patients with gastric lesions often require gastroscopy [10-12]. The gastrointestinal mucosa can be directly observed. At the same time, some diseased tissue and cells can be removed. However, due to different growth patterns and irregular tumor morphology, gastroscopy may reveal ulcers and erosion, and there is limited pathologic material to evaluate tumor and biological behavior as a whole.

Original data iterative reconstruction algorithm sinogram iterative reconstruction algorithm (SAFIRE) is based on original data [13-15]. By iterating in projected data space and image space, the original data is repeatedly inserted for iterative reconstruction. The purpose is to reduce the noise of the image and to maintain the sharpness of the image. With the increase of iteration level, the noise is further reduced. In recent years, many researchers studied the application of SAFIRE in the imaging diagnosis of chest diseases. It was found that SAFIRE can improve image quality to a certain extent compared with the filtered back projection (FBP) reconstruction algorithm [16]. Therefore, the clinical application of SAFIRE technology can meet the clinical diagnostic requirements with the same image quality, while reducing the radiation dose of the patient by reducing the voltage of the tube. Some studies analyzed the application of SAFIRE in abdominal CT examination [17]. However, the application of SAFIRE in the abdominal examination is mainly focused on solid organs, and there are still few studies on its application in cavity organ examination.

In summary, this study adopted CT imaging based on the SAFIRE algorithm to diagnose the clinical staging of gastric cancer and used the pathological diagnosis result as the gold standard to evaluate its diagnostic effect. The 1 to 5 levels of the AFIRE algorithm were compared with the FBP reconstruction algorithm, so as to explore the application value of the AFIRE algorithm in preoperative staging scans of gastric cancer and provide more and more effective clinical methods for the staging diagnosis of clinical gastric cancer patients.

\section{Methods}

2.1. Research Objects. From August 2018 to April 2020, 59 patients with gastric cancer in the hospital were recruited as the research objects. There were 38 males and 21 females, ranging in age from 28 to 81 years, with an average age of $61 \pm 11.09$ years. CT scan was performed on all patients with gastric cancer, SAFIRE was used for treatment in clinical staging diagnosis of gastric cancer, and pathological changes of the normal stomach were studied on each patient, with pathological diagnosis results as the gold standard. According to histopathological classification, there were 14 cases of signet-ring cell carcinoma, 39 cases of signet-ring cell adenocarcinoma, 6 cases of signet-ring cell carcinoma with partial adenocarcinoma, 6 cases of adenocarcinoma with partial signet-ring cell carcinoma, 6 cases of adenocarcinoma with partial signet-ring cell carcinoma, 29 cases of distal gastric carcinoma, 9 cases of gastric antrum carcinoma, 1 case of gastric fundus carcinoma, 3 cases of total gastric carcinoma, 13 cases of gastric body and angle carcinoma, and 4 cases of gastric cardia carcinoma. The small lesion was about $1.5 \mathrm{~cm} \times 1.5 \mathrm{~cm}$, and the large lesion mostly involved the whole stomach. The main clinical symptoms were abdominal pain, anorexia, epigastrium discomfort, anemia, vomiting, hematemesis and black feces, epigastrium mass, emaciation, etc.

\subsection{Inclusion and Exclusion Criteria. Inclusion criteria are as} follows: (i) all patients underwent CT examination within two weeks before surgery, the gastric cavity was full of gas, and lesion sites were clear; (ii) postoperative pathology confirmed that all patients had gastric cancer; and (iii) all the lesions were preoperative lesions without chemotherapy or radiotherapy.

Exclusion criteria are as follows: (i) those who had other stomach diseases, such as gastritis, gastric ulcer, or other tumors, accompanied by other stomach diseases; (ii) the gastric cavity was poorly filled, and the lesion was not clearly displayed; and (iii) the pathological changes were mainly caused by postoperative human intervention, radiotherapy, and chemotherapy.

\subsection{Iterative Reconstruction Algorithm}

2.3.1. The Basic Idea of Iterative Reconstruction. The iterative algorithm first initializes the FBP algorithm with weights and then uses two different adjustment loops, one of which iterates the original data in space to eliminate the noise in the original data domain. It adds many steps to verify the original data and uses the detected deviations to weight FBP to generate updated images. The number of iterations of this loop depends on the scan type. The second cycle is used to calibrate the image space. Based on the statistical optimization process, the propagation path of noise in the original data is also used. The image calibration is constantly compared with the original image, and the repeated processing engineering also depends on the specific scan type. The end node is determined by the user's definition of image quality. Due to the introduction of iteration into the original data field, the ratio of dose reduction is greatly improved compared with the scale image. Under the same dose, the image quality becomes more accurate, but higher computer resources are required. In the case of improved computer hardware configuration, the hybrid iterative reconstruction speed reaches 20 images per second.

\subsubsection{Specific Process of Iterative Reconstruction Algorithm.} The iterative reconstruction algorithm needs to solve the physical model of the system, the statistical model of the 
measurement, and the necessary evaluation function and the normal term. The model-based iterative algorithm takes into account the system characteristics and the various dimensions of image reconstruction, which minimizes the image noise. When a system model is constructed, a linear approximation method is usually used, and the X-ray emitted from the detector receives an approximate processing point, which is expressed as follows:

$$
\begin{aligned}
I_{i} & \approx I_{0} \exp \left(-\sum_{j=1}^{N} a_{i j} X_{j}\right), \\
\left(a_{i j}\right) & =\int \text { source } \int \text { detector } \int \psi \overrightarrow{\left(p_{s}, p_{d}\right)} b_{j} \overrightarrow{(r)} d \overrightarrow{d p_{s}} d \overrightarrow{p_{d}} .
\end{aligned}
$$

The basic equation is that one pixel calculates $x$ from the actual measured value $y$.

$$
\overrightarrow{f(r)} \approx \vec{f}(\vec{r})=\sum_{j=1}^{N} X_{j} b_{j}(\vec{r}) .
$$

The processing of forward and backward projection pairs is an indispensable iterative process in the model. For the projection from the image domain to the projection domain, it is also called forward projection. The expression is as follows:

$$
\bar{y}=A X, \overline{y_{i}}=\sum_{j=1}^{N} a_{i j}, X_{j}=[A x] .
$$

The representation of the back projection from the projection domain to the image domain is as follows:

$$
\begin{aligned}
& z=A^{\prime} y, z_{j}=\sum_{i=1}^{M} a_{i j} y_{i}, \\
& \widehat{X}=\arg \min \|y-A X\|^{2}=\left[A^{\prime} A\right]^{-1} A^{\prime} y .
\end{aligned}
$$

It is assumed that the X-ray is a monochromatic source in the noise statistical model, without considering the scattering effect, and the expression equation of the original noise measurement is as follows:

$$
\bar{I}_{i}\left[(A X)_{i}\right]=I_{0}{ }^{e}-\sum_{j=1}^{N} a_{i j} x_{j} .
$$

Linear logarithmic model is expressed as follows:

$$
y_{i} \cong-\log \left(\frac{I_{i}}{I_{O}}\right) \simeq[A x]_{i}+\varepsilon_{i} .
$$

Hypothetical Gaussian noise mode is $\varepsilon_{i} \sim N\left(0, \delta_{i}^{2}\right)$.

The evaluation function of this model consists of two parts of data mismatch item and normal item. The minimum evaluation function of the reconstructed image $\hat{x}$ is as follows:

$$
\begin{aligned}
\widehat{X} & =\arg \min \psi(x) ; x \geq 0, \\
\psi(x) & =\text { Datamismatch }(y, A X)+\beta \text { Regularizer }(x), \\
\text { Datamismatch }(y, A x) & =-L(x ; y)=-\log (y \mid x)=\sum_{i=1}^{M}-\log p\left(y_{i} \mid x\right), \\
-L(x ; y) & =\sum_{i=1}^{M} W_{i} \frac{1}{2}\left(y_{i}-[A x]_{i}\right)^{2} \\
& =\frac{1}{2}\left(y-A X^{\prime}\right) W(y-A X), W_{i}=\frac{1}{\sigma_{i}^{2}}, \\
\operatorname{Regularizer}(X) & -\sum_{j=1}^{N} \frac{1}{2} \sum_{k \varepsilon N_{j}} \psi\left(X_{i}-X_{K}\right) .
\end{aligned}
$$

The parabolic equation of the potential function $\psi(t)$ in the upper, lower, left, and right neighborhoods of the $j$-th pixel $N_{j}$ is expressed as follows:

$$
\psi(t)=\sqrt{1+(t / \delta)}^{2} .
$$

The carrier-to-noise ratio (CNR) and signal-to-noise ratio (SNR) were used for the evaluation of the algorithm.

\subsection{Apparatus and Methods}

2.4.1. CT Scan. The tube voltages of the two tubes were $80 \mathrm{kVp}$ and $140 \mathrm{kVp}$, respectively, and the scanning layer thickness was $5 \mathrm{~mm}$. The traditional FBP algorithm (B30) and iris algorithm (ib30) were used in the construction. The high-pressure syringe contrast agent iodohexanol (300 MGI/M1) was injected into the upper abdomen at a flow rate of $3.0 \mathrm{~mL} / \mathrm{s}$ and a body weight of $2 \mathrm{~mL} / \mathrm{kg}$. The dual-energy scan was performed at $25 \mathrm{~s}$ and $70 \mathrm{~s}$ after high-pressure injection. The upper boundary of the scan was the apex of the diaphragm, and the lower boundary was the liver and stomach.

2.4.2. FBP Image Processing. The "View" software measured the images obtained by dual-energy scanning. CT value of gastric cancer and normal gastric wall in the arterial phase and venous phase was measured, and the difference (act value) of the CT value of gastric cancer and the normal gastric wall was calculated. The same method was used to measure the CT value and noise of gastric cancer and liver arterial phase and venous phase in $\mathrm{fbp}-\mathrm{de}, \mathrm{fbp}-80 \mathrm{kVp}$, and iris- $80 \mathrm{kVp}$ images.

2.4.3. Gastroscopy Examination. Japanese Olympus grf- 260 gastroscopy was selected for examination. Client was placed in the left supine position on an empty stomach. Oral anesthesia was administered 15 minutes before examination. 
Then, the gastroscope was inserted from the patient's throat into the stomach. The patient's lesion was observed by gastroscope, and the location and size of the tumor were recorded.

2.5. CT Scan in the Classification of Gastric Cancer Patients. In this experiment, LR was used for classification. LR is a commonly used machine learning method to estimate the likelihood of something and classify it. LR processing data are continuous, discrete, and mixed.

$80 \%$ of the computer-generated data of 47 patients with gastric cancer were randomly included, and 11 cases of signet-ring cell carcinoma and 31 cases of adenocarcinoma were divided into the training group $(n=42)$. Eight patients with adenocarcinoma and three with signet-ring cell carcinoma (out of 11) were assigned to the validation group. According to clinical needs, the features after dimension reduction were used for relevant research and analysis (Figure 1).

2.6. Statistical Methods. SPSS 19.0 was employed for data statistics and analysis. Mean \pm standard deviation $(\bar{x} \pm s)$ was how measurement data were expressed. Under the same scanning conditions, the SAFIRE 1-5 images and the FBP reconstruction algorithm images were compared in pairs, and the numerical variables were analyzed by one-way analysis of variance. Statistical analysis was performed with $P=0.05$ as the test standard.

\section{Results}

3.1. Denoising Effect of Iterative Reconstruction Algorithm. Figure 2 shows the comparison of CNR and SNR results of $\mathrm{CT}$ images before and after processing by the iterative reconstruction algorithm under the same radiation dose. It illustrated that the CNR (0.979) and SNR (0.967) after processing were significantly higher than those before $(0.781,0.744)(P<0.05)$.

3.2. Comparison of CT Values of Each Group under the Guidance of Iterative Reconstruction Algorithm. A single analysis of variance was adopted, and the results showed that there was no significant difference between the FBP algorithm image and the SAFIRE (level 1 to 5) image in the measured average CT values of each part $(P>0.05)$ (Figures 3 and 4 ). One-way analysis of variance was performed to compare six groups of subjective image quality scores. The results showed that the FBP reconstructed image had the highest score, while the SAFIRE image gradually decreased as its grade increased. There was no significant difference in the image scoring results between the FBP algorithm and S1, S2, and S3 ( $P$ values were $0.54,0.31$, respectively), but there was a significant difference compared with the results of S4 5 $(P<0.05)$. Therefore, it was considered that the differences in the display of lesions and depth of invasion between the two groups of images were not statistically significant.

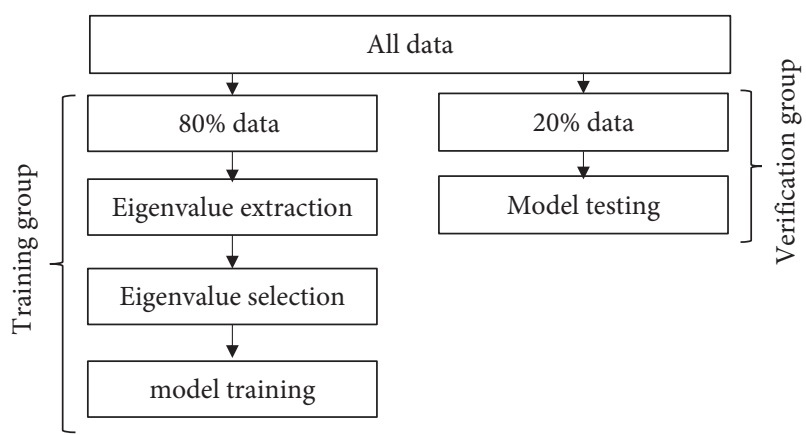

FIgURE 1: Statistical analysis process.

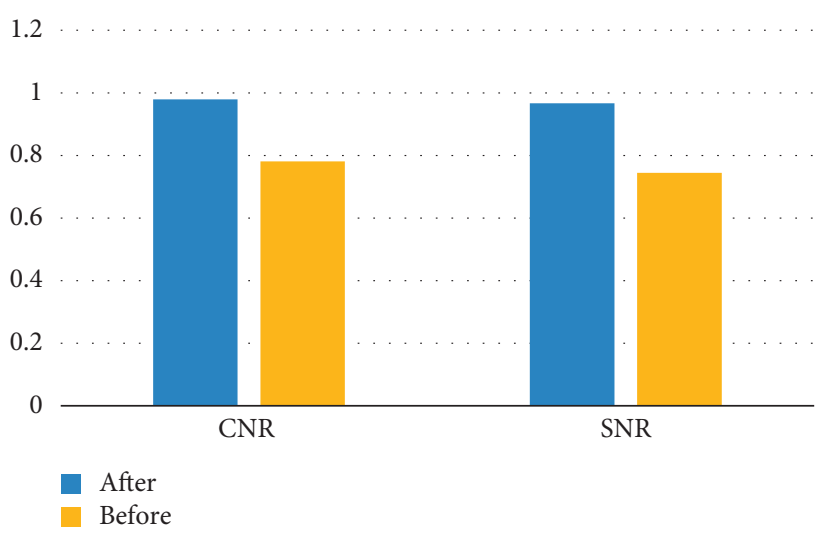

Figure 2: Comparison of denoising effects of iterative reconstruction algorithms.

3.3. The Feasibility Study of CT Plain Scan Imaging Omics in the Diagnosis of Gastric Cancer under the Guidance of Iterative Reconstruction Algorithm. KNN classifier was used for training, and ROC analysis curve was used to illustrate the prediction performance of radiation characteristics. The model evaluation index (area under the curve, AUC) of the training group was 0.999 , and the AUC of the validation group was 0.958 . The sensitivity and specificity were both above $90 \%$, and the difference was not statistically significant $(P>0.05)$. The research results can provide a reference for the establishment of gastric cancer imaging research database, assist clinical work, and improve the characteristic curve of the KNN classification method (Figure 5).

3.4. The Feasibility Study of CT Plain Scan Imaging Omics in the Pathological Classification of Gastric Cancer. In this study, the gold standard of postoperative pathology was used to study the classification of patients with gastric cancer. Logistic regression (LR) classifier was used for training, and ROC analysis curve was used to interpret the prediction of radioactive characteristics. The AUC of the training set was 0.969 , and the AUC of the verification set was 0.8599 . Signetring cell carcinoma had medium sensitivity and high specificity, and adenocarcinoma had high sensitivity and medium specificity. The characteristic curve of the LR method was established to provide a reference for differential diagnosis of gastric adenocarcinoma and signet-ring cell carcinoma. 


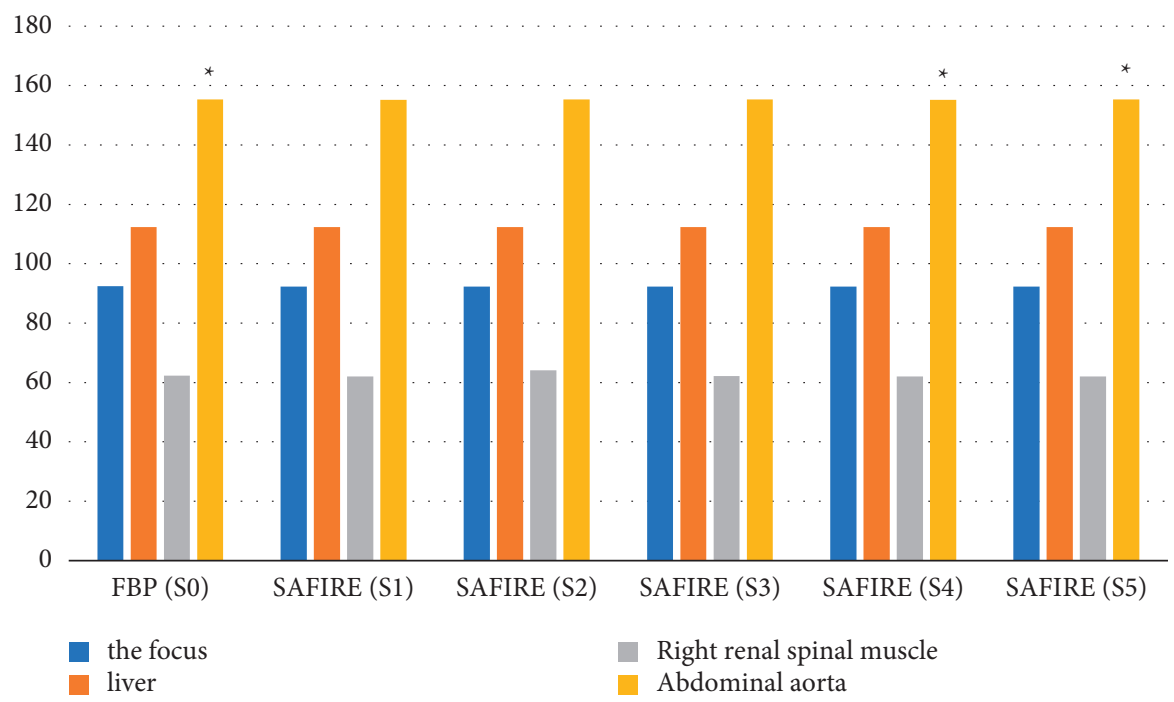

Figure 3: Comparison of average CT values of six groups. Note: ${ }^{*}$ suggests that the difference was statistically obvious $(P<0.05)$.

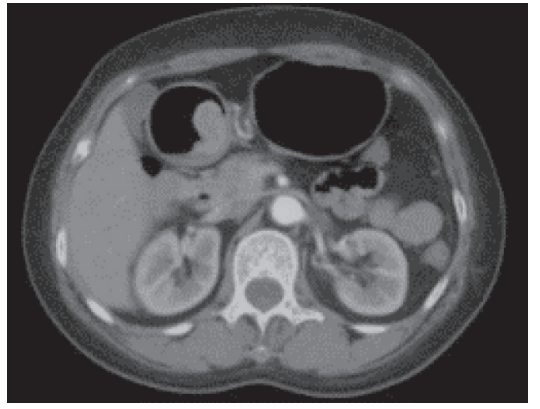

(a)

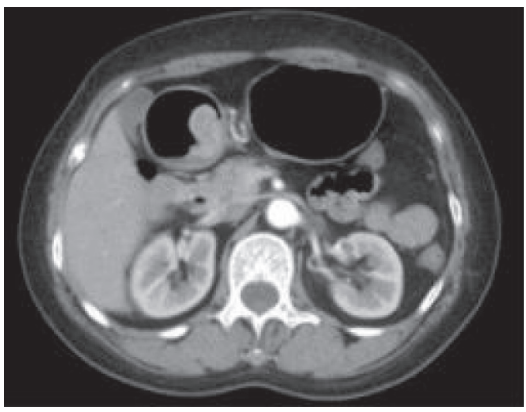

(b)

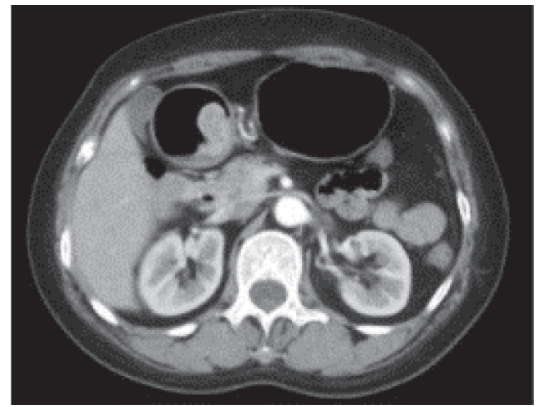

(c)

FIGURE 4: Results before and after reconstruction of CT images of gastric cancer. (a) Reference image; (b) FBP reconstruction result; (c) iterative reconstruction result.

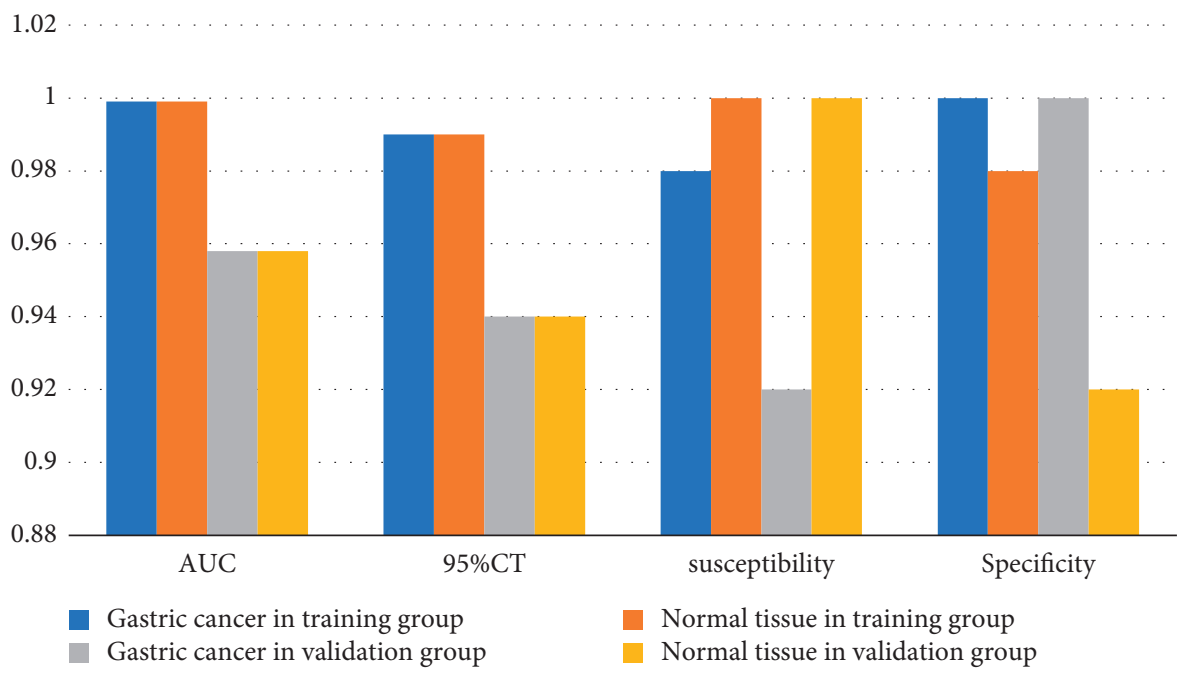

FIgURE 5: ROC curve analysis of KNN classifier.

To increase the differential diagnosis of gastric adenocarcinoma and sigmoid ring cell carcinoma, two of the most valuable features were selected from the extracted eight eigenvalues for statistical analysis, including differential entropy and skewness. The diagnostic efficiency was moderate and was of clinical research value, which can provide reference significance for pathological classification and diagnosis of gastric cancer (Figure 6). 


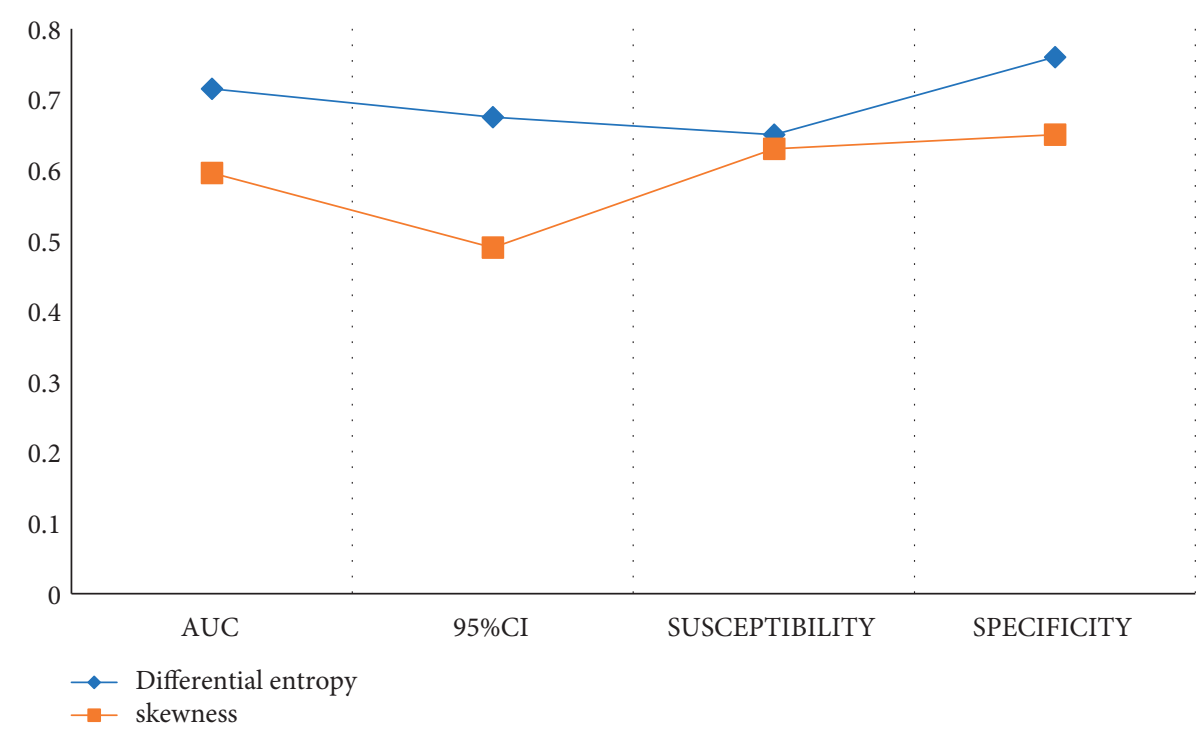

Figure 6: Diagnostic efficiency of differential entropy and skewness.

\subsection{Comparison of CT and Gastroscopy Detection Outcomes.} The difference between the probability of detecting gastric cancer by CT and gastroscopy was compared, and it was found that the detection rate of CT was $93 \%$, while the detection rate of gastroscopy was $95 \%$. After comparison, it was found that there was no significant difference in the detection rate of gastric cancer between spiral CT and gastroscopy $(P>0.05$, Figure 7$)$.

3.6. Judgment of T Staging before CT Operation. The consistency between preoperative CT staging and pathological diagnosis staging was compared. The results showed that CT staging and pathological diagnosis had the largest number of patients in T3 stage, followed by T2 stage. There were two patients diagnosed as T2 stage by $\mathrm{CT}$, and the pathological diagnosis was determined as T3 stage. Two patients were diagnosed as T3 stage with $\mathrm{CT}$, and the pathological diagnosis was determined as T4 stage. Therefore, the preoperative CT staging kappa value was consistent with the postoperative pathological diagnosis $0.882(P<0.05$, Figure 8). The effect of CT preoperative staging of gastric cancer was consistent with the results of pathological diagnosis and staging.

\section{Discussion}

Gastric cancer is one of the common malignant tumors in China [18]. The most commonly used histological classification of gastric cancer is the WHO histological classification (1990 edition), which is classified into adenocarcinoma, squamous cell carcinoma, adenosquamous carcinoma, and undifferentiated carcinoma. Adenocarcinomas are subdivided into papillary, tubular, mucinous, signet-ring cell, and poorly differentiated adenocarcinomas. In this study, papillary adenocarcinoma and highly/moderately differentiated tubular adenocarcinoma were classified as the group with a good prognosis, while poorly differentiated

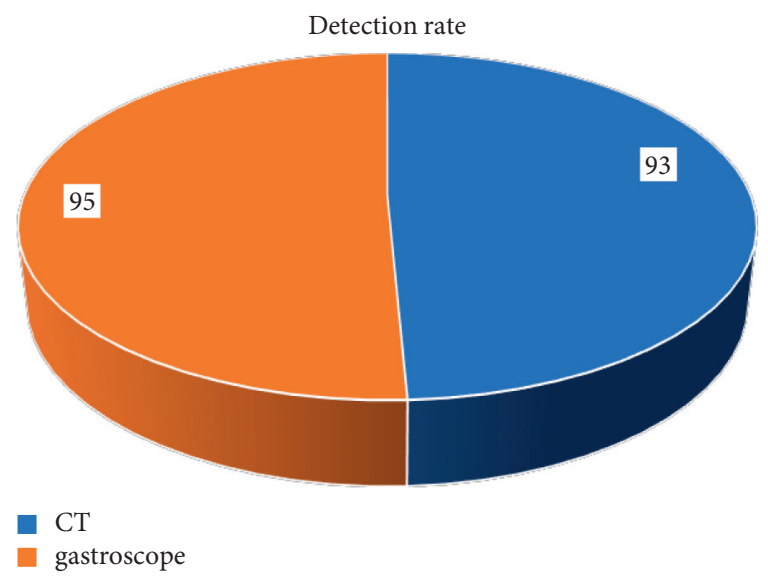

FIGURE 7: Comparison of CT and gastroscopy detection outcomes.

adenocarcinoma, mucinous adenocarcinoma, and signetring cell carcinoma were classified as the group with a poor prognosis, according to the results of literature on the structure of the imaging omics model [19-21]. Some researchers reported that this type of gastric cancer was classified into differentiated and undifferentiated gastric cancer [22]. The above are the common types of gastric cancer, while other rare types only account for $5 \%$ of gastric cancer $[23,24]$.

At present, the incidence and death rate of gastric cancer in China are both higher than the world average, ranking among the top 20. Accurate preoperative staging of gastric cancer patients is of great significance for the selection of appropriate treatment and the evaluation of prognosis. Especially, with the development of new technologies such as early gastric cancer endoscopic mucosal resection, laparoscopic radical gastrectomy, and preoperative neoadjuvant chemotherapy, accurate preoperative staging of gastric cancer becomes very important. In most patients with advanced gastric cancer, the application of adjuvant and neoadjuvant chemotherapy and the combined 


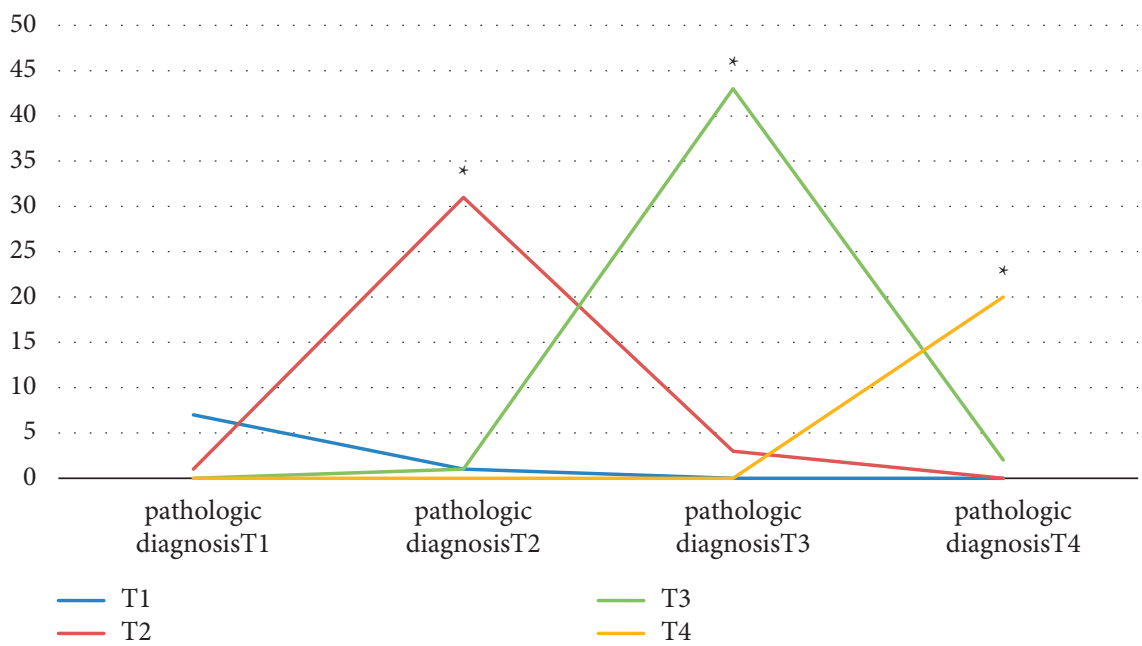

Figure 8: Judgment of T staging before CT operation. Note: ${ }^{*}$ suggests that the difference was statistically obvious $(P<0.05)$.

application of radiotherapy can enhance the survival rate of patients and improve the prognosis and rehabilitation $[25,26]$. Among the many tests for preoperative staging of gastric cancer, the enhanced CT scan is fast. In addition, it can display the relationship between the lesion and adjacent tissues and organs, and whether there is distant metastasis, which makes its clinical application more and more widely. Chen and Liu (2016) [27] reported that CT texture analysis was used to predict the pathological characteristics of gastric cancer tissues and found that the tumor tissues were related to Lauren classification. In addition, the average attenuation and maximum attenuation of portal vein CT images of diffuse gastric cancer were significantly higher than those of intestinal gastric cancer. The texture parameters of portal vein $C T$ images were negatively correlated with the differentiation degree of gastric cancer. Data from 107 patients with gastric cancer were analyzed. The computer software reads the segmented region of the gastric cancer lesion from the region of interest, including mean attenuation, and automatically sets the distribution of the eight features. The relationship between CT tissue and histopathologic features was then evaluated by statistical methods. It was not difficult to see from the data obtained from the imaging results that the model was of the best performance in predicting the different prognostic histological types of the three different types of gastric cancer. According to the prediction model, gastric cancer in younger age and more histological types was often classified as gastric cancer with poor prognosis and higher degree of malignancy. This was the same as the report of MacDougall et al. (2016) [28]. The younger the cancer cells were, the more active the metabolism was, the faster the progression was, and the higher the degree of malignancy was in the early stage of gastric cancer. In addition, it was necessary for young and middle-aged people to have gastroscopy. Moreover, the prediction model found that the tissue type of gastric cancer metastasis was more likely to have a poor prognosis, which was consistent with the clinical reality. However, no subdivision of metastasis, local metastasis, and distant metastasis was found, and further study was needed.
Based on the use of the SAFIRE algorithm, the effect of the algorithm used in this study was also analyzed. The results showed that the CNR (0.979) and SNR (0.967) of CT images after the algorithm processing were significantly higher than those before $(0.781,0.744)(P<0.05)$. It showed that the SAFIRE algorithm had a certain effect in the processing of CT images, which was consistent with the research results of Qurashi et al. (2018) [29]. Comparison of the application of the SAFIRE algorithm and FBP algorithm revealed that there was no significant difference in CT value between the FBP algorithm and S1, S2, and S3 $(P>0.05)$. But S4 S5 were significantly lower than the FBP algorithm, indicating that the higher the SAFIRE algorithm level, the effect was close to the CT image processing effect of the FBP algorithm. The research results of Scharf et al. (2017) [30] also concluded that the higher the level of the SAFIRE algorithm, the better the image of the processing effect.

\section{Conclusion}

In this study, it explored the application value of CT imaging under the guidance of the sinogram-affirmed iterative reconstruction algorithm (SAFIRE) in the clinical staging of gastric cancer. The results showed that CT images guided by SAFIRE algorithms can objectively and noninvasively assess tumor asymmetry, make reasonable staging diagnosis of gastric cancer, and provide useful information for clinicians to develop high-quality individualized treatment plans. However, this study only selected portal vein CT images for feature extraction and only selected the largest layer of lesions on the CT axis may lead to partial sampling errors and insufficient external verification, and all these issues required further research. However, it fully demonstrated the good application prospects of intelligent algorithms in the field of medical imaging in this study.

\section{Data Availability}

The data used to support the findings of this study are available from the corresponding author upon request. 


\section{Conflicts of Interest}

The authors declare no conflicts of interest.

\section{Authors' Contributions}

Chunfang Zhou and Shufang Tian contributed equally to this work.

\section{References}

[1] J.-P. Gao, W. Xu, W.-T. Liu, M. Yan, and Z.-G. Zhu, “Tumor heterogeneity of gastric cancer: from the perspective of tumor-initiating cell," World Journal of Gastroenterology, vol. 24, no. 24, pp. 2567-2581, 2018.

[2] W.-M. Kang, Q.-B. Meng, J.-C. Yu, Z.-Q. Ma, and Z.-T. Li, "Factors associated with early recurrence after curative surgery for gastric cancer," World Journal of Gastroenterology, vol. 21, no. 19, pp. 5934-5940, 2015.

[3] J. M. Niesten, I. C. van der Schaaf, P. C. Vos, M. J. Willemink, and B. K. Velthuis, "Improving head and neck CTA with hybrid and model-based iterative reconstruction techniques," Clinical Radiology, vol. 70, no. 11, pp. 1252-1259, 2015.

[4] R. J. Gillies, P. E. Kinahan, and H. Hricak, "Radiomics: images are more than pictures, they are data," Radiology, vol. 278, no. 2, pp. 563-577, 2016.

[5] H. Peng, D. Dong, M.-J. Fang et al., "Prognostic value of deep learning PET/CT-Based radiomics: potential role for future individual induction chemotherapy in advanced nasopharyngeal carcinoma," Clinical Cancer Research, vol. 25, no. 14, pp. 4271-4279, 2019.

[6] J. Song, J. Shi, D. Dong et al., "A new approach to predict progression-free survival in stage IV EGFR-mutant NSCLC patients with EGFR-TKI therapy," Clinical Cancer Research, vol. 24, no. 15, pp. 3583-3592, 2018.

[7] Z. Ma, M. Fang, Y. Huang et al., "CT-based radiomics signature for differentiating Borrmann type IV gastric cancer from primary gastric lymphoma," European Journal of Radiology, vol. 91, pp. 142-147, 2017.

[8] D. Dong, L. Tang, Z.-Y. Li et al., "Development and validation of an individualized nomogram to identify occult peritoneal metastasis in patients with advanced gastric cancer," Annals of Oncology, vol. 30, no. 3, pp. 431-438, 2019.

[9] S. W. T. Ho and P. Tan, "Dissection of gastric cancer heterogeneity for precision oncology," Cancer Science, vol. 110, no. 11 , pp. 3405-3414, 2019.

[10] S. Liu, S. Liu, C. Ji et al., "Application of CT texture analysis in predicting histopathological characteristics of gastric cancers," European Radiology, vol. 27, no. 12, pp. 4951-4959, 2017.

[11] H. Y. Kim, Y. H. Kim, G. Yun, W. Chang, Y. J. Lee, and B. Kim, "Could texture features from preoperative CT image be used for predicting occult peritoneal carcinomatosis in patients with advanced gastric cancer?” PLoS One, vol. 13, no. 3, Article ID e0194755, 2018.

[12] J. G. Fletcher, L. Yu, Z. Li et al., "Observer performance in the detection and classification of malignant hepatic nodules and masses with CT image-space denoising and iterative reconstruction," Radiology, vol. 276, no. 2, pp. 465-478, 2015.

[13] L.-M. Wei, S. Cao, W.-D. Yu, Y.-L. Liu, and J.-T. Wang, "Overexpression of CX3CR1 is associated with cellular metastasis, proliferation and survival in gastric cancer," Oncology Reports, vol. 33, no. 2, pp. 615-624, 2015.
[14] Y. Kim, Y. K. Kim, B. E. Lee et al., "Ultra-low-dose CT of the thorax using iterative reconstruction: evaluation of image quality and radiation dose reduction," American Journal of Roentgenology, vol. 204, no. 6, pp. 1197-1202, 2015.

[15] S. Wu, J. Zheng, Y. Li et al., "A radiomics nomogram for the preoperative prediction of lymph node metastasis in bladder cancer," Clinical Cancer Research, vol. 23, no. 22, pp. 6904-6911, 2017.

[16] R. T. H. M. Larue, G. Defraene, D. De Ruysscher, P. Lambin, and W. van Elmpt, "Quantitative radiomics studies for tissue characterization: a review of technology and methodological procedures," British Journal of Radiology, vol. 90, no. 1070, Article ID 20160665, 2017.

[17] M. Avanzo, J. Stancanello, and I. El Naqa, "Beyond imaging: the promise of radiomics," Physica Medica, vol. 38, pp. 122-139, 2017.

[18] B. Yang, M. Yan, Z. Yan, C. Zhu, D. Xu, and F. Dong, "Segmentation and classification of thyroid follicular neoplasm using cascaded convolutional neural network," Physics in Medicine and Biology, vol. 65, no. 24, Article ID 245040, 2020.

[19] Y.-q. Huang, C.-h. Liang, L. He et al., "Development and validation of a radiomics nomogram for preoperative prediction of lymph node metastasis in colorectal cancer," Journal of Clinical Oncology, vol. 34, no. 18, pp. 2157-2164, 2016.

[20] F. Orlhac, B. Thézé, M. Soussan, R. Boisgard, and I. Buvat, "Multiscale texture analysis: from 18F-fdg PET images to histologic images," Journal of Nuclear Medicine, vol. 57, no. 11, pp. 1823-1828, 2016.

[21] M. Hatt, F. Tixier, D. Visvikis, and C. Cheze Le Rest, "Radiomics in PET/CT: more than meets the eye?" Journal of Nuclear Medicine, vol. 58, no. 3, pp. 365-366, 2017.

[22] Z. Zhou, M. Folkert, N. Cannon et al., "Predicting distant failure in early stage NSCLC treated with SBRT using clinical parameters," Radiotherapy \& Oncology, vol. 119, no. 3, pp. 501-504, 2016.

[23] S. Liu, H. Shi, C. Ji et al., "Preoperative CT texture analysis of gastric cancer: correlations with postoperative TNM staging," Clinical Radiology, vol. 73, no. 8, pp. e1-756, e9, 2018.

[24] F. Schaller, M. Sedlmair, R. Raupach, M. Uder, and M. Lell, "Noise reduction in abdominal computed tomography applying iterative reconstruction (ADMIRE)," Academic Radiology, vol. 23, no. 10, pp. 1230-1238, 2016.

[25] L. Sukupova, "What does CTDIVOL mean and is this parameter suitable for the determination of patients' doses?" Ceská Radiologie, vol. 69, no. 3, pp. 194-200, 2015.

[26] P. H. Jeon, W. H. Lee, S. S. Jeon, and H. J. Kim, "Evaluation of imaging characteristics in CTDI phantom size on contrast imaging," Medical Imaging: Physics of Medical Imaging. International Society for Optics and Photonics, vol. 9412, 2015.

[27] F. L. Chen and A. S. Liu, "Clinical application of ECG gated CTA in evaluation of acute aortic dissection," CT Theory and Applications, vol. 25, no. 5, pp. 547-554, 2016.

[28] R. D. MacDougall, P. L. Kleinman, and M. J. Callahan, "Sizebased protocol optimization using automatic tube current modulation and automatic $\mathrm{kV}$ selection in computed tomography," Journal of Applied Clinical Medical Physics, vol. 17, no. 1, pp. 328-341, 2016.

[29] A. Qurashi, L. Rainford, A. Ajlan et al., "Optimal abdominal CT protocol for obese patients,” Radiography, vol. 24, no. 1, pp. el-e12, 2018.

[30] M. Scharf, S. Brendel, K. Melzer et al., "Image quality, diagnostic accuracy, and potential for radiation dose reduction in thoracoabdominal CT, using Sinogram Affirmed Iterative Reconstruction (SAFIRE) technique in a longitudinal study," PLoS One, vol. 12, no. 7, Article ID e0180302, 2017. 